\title{
Water and health in mining settings in sub-Saharan Africa: A mixed methods geospatial visualization
}

\author{
Andrea Leuenberger, ${ }^{1,2}$ Dominik Dietler, ${ }^{1,2}$ Isaac Lyatuu, ${ }^{1-3}$ Andrea Farnham, ${ }^{1,2}$ \\ Fadhila Kihwele, ${ }^{3}$ Fritz Brugger, ${ }^{4}$ Mirko S. Winkler ${ }^{1,2}$ \\ ${ }^{1}$ Swiss Tropical and Public Health Institute, Basel, Switzerland; ${ }^{2}$ University of Basel, Basel, Switzerland; \\ ${ }^{3}$ Ifakara Health Institute, Dar es Salaam, Tanzania; ${ }^{4}$ Department of Humanities, Social- and Political \\ Sciences, Swiss Federal Institute of Technology (ETH), Zurich, Switzerland
}

\begin{abstract}
Industrial mining transforms local landscapes, including important health determinants like clean water and sanitation. In this paper, we combined macro-level quantitative and micro-level qualitative data to show how mining projects affect water infrastructures and ultimately the health of affected communities. Although we observed a positive trend of water infrastructure in mining settings, surrounding communities are also characterized by water scarcity and degradation of water quality. The video at the core of this publication showcases inter-linkages of the findings obtained at both the macro- and the micro-levels, embedding our results in a geospatial context. While mining projects can have positive impacts on the development of local water infrastructure, improved management of negative impacts of mining projects is needed for promoting 'Good health and well-being' and 'Clean water and sanitation' as promulgated by the Sustainable Development Goals of the 2030 Agenda.
\end{abstract}

\footnotetext{
Correspondence: Andrea Leuenberger and Dominik Dietler, Swiss Tropical and Public Health Institute, and University of Basel, P.O. Box, CH-4003, Basel, Switzerland.

E-mail: andrea.leuenberger@swisstph.ch; dominik.dietler@swisstph.ch
}

Key words: Extractive industries; health; impact assessment; mixed methods; WASH.

Contributions: Andrea Leuenberger and Dominik Dietler contributed equally as first authors.

Video clip: https://www.youtube.com/watch?v=4EcJ2rDQEuU

Received for publication: 7 December 2020.

Revision received: 8 March 2021.

Accepted for publication: 8 March 2021.

(C) Copyright: the Author(s), 2021

Licensee PAGEPress, Italy

Geospatial Health 2021; 16:965

doi:10.4081/gh.2021.965

This article is distributed under the terms of the Creative Commons Attribution Noncommercial License (CC BY-NC 4.0) which permits any noncommercial use, distribution, and reproduction in any medium, provided the original author(s) and source are credited.

\section{Introduction}

Water security is a key public health issue of the $21^{\text {st }}$ century (Boretti \& Rosa, 2019). The importance of water is also reflected in the 2030 Agenda for Sustainable Development, aiming for ' universal and equitable access to safe and affordable drinking water for all' under the sixth Sustainable Development Goal (SDG) (United Nations, 2015). Access to water is intrinsically linked to the attainment of good health and well-being - prominently featured in the 2030 Agenda for Sustainable Development under SDG 3. Achieving these ambitious goals requires collaboration between different sectors - including extractive industries.

Large resource extraction projects, such as industrial mines, are an important economic driver in many of the countries where water scarcity is an acute problem (Admiraal et al., 2017). The implementation of mining projects can have a positive effect on local water infrastructures through direct investments and local economic growth (von der Goltz \& Barnwal, 2019). On the other hand, mining activities can also increase the demand of local water resources or cause water pollution, and therefore, negatively affect access to clean water in local communities (Kemp et al., 2010; Schrecker et al., 2018). Rapid in-migration of job seekers to often remote and rural mining areas can additionally strain the often weak water infrastructures and limited water resources (Pelders \& Nelson, 2018). Hence, as a key determinant of health, changes of local water resources are closely interlinked with the health and well-being of affected populations (Marcantonio et al., 2021).

To assess these positive and negative impacts of large infrastructure projects on water resources and health, environmental impact assessments (EIAs) are conducted in virtually all countries in the world (Morgan, 2012). EIAs aim to determine how to mitigate potential negative environmental impacts along with maximising potential benefits for society prior to project development (Morgan, 2012). Hence, impact assessment can serve as a tool to engage mining companies toward the attainment of the 2030 Agenda for Sustainable Development (Winkler et al., 2020).

To inform impact assessment practice, it is important to better understand in which direction, and to what extent, water infrastructures are impacted in mining settings and ultimately how this affects the health of surrounding communities. To date, studies on the impacts of mining projects on water often focus on environmental effects, while inclusion of health implications for surrounding communities remains weak. Particularly, research triangulating qualitative and quantitative data at both the supra-national and local levels is scarce. In this paper, we utilized a mixed methods approach to better understand how mining affects access 
to safe and affordable water and how this in turn impacts the health status of local communities in sub-Saharan Africa.

\section{Materials and methods}

In the frame of a multi-country research project, we combined quantitative and qualitative data (Figure 1) (Farnham et al., 2020; Winkler et al., 2020). Quantitative data were used to analyse the trends in access to different water sources (i.e. piped water, wells, surface water) from a macro-level perspective. Based on qualitative data, water reliability and quality were described on a microlevel as perceived by affected community members. Together with insights from EIA reports, we triangulated all our findings to present a comprehensive overview of the complex and dynamic relationship between mining projects, water infrastructures and community health. The video at the core of this publication allowed us to embed our results in a geospatial context and to showcase linkages of the findings obtained at different levels.

\section{Macro-level: regional trends with regard to water infrastructures around industrial mining sites}

For quantifying the mining impacts on water infrastructures at the national and supra-national level, data from all Demographic and Health Surveys (DHS) conducted in 34 sub-Saharan African countries were combined (Dietler et al., 2021, undergoing peer review). DHS data are generated through repeated standardized household surveys, allowing for comparison of access to water in mining areas both across and within countries over time.

The Standard \& Poor's Global Market Intelligence Mining Database featuring all large mines in Africa was used to select the more than 189,992 DHS households located within the distance of $100 \mathrm{~km}$ from an industrial mine (Dietler et al., 2021, undergoing peer review). The households were categorized based on their distance to the mining site. Households located within a radius of 5 $\mathrm{km}$ from a mining project were classified as impacted. Households at a distance of 50-100 km from the closest mine were used for comparison. Households located in larger cities were excluded from analysis. Using multi-level multinomial logistic regression models, the impact of proximity to a mine on access to water infrastructures and diarrheal incidence in the two weeks prior to the survey was analysed.

\section{Micro-level: perceived impacts on water and health in communities around industrial mining sites}

For a better understanding of the impacts of mining companies on local water infrastructures, we collected primary data in two industrial gold mining sites in north-western Tanzania; more precisely, we engaged with communities around the Buzwagi and Bulyanhulu Gold mine. Rooted in the qualitative geographic information systems (Qualitative GIS) approach (Lechner et al., 2019), we first conducted focus group discussions (FGDs), followed by a thematic analysis to extract statements related to water. Subsequently, we geo-referenced the water sources mentioned during the discussions (Leuenberger et al., 2021, undergoing peer review).

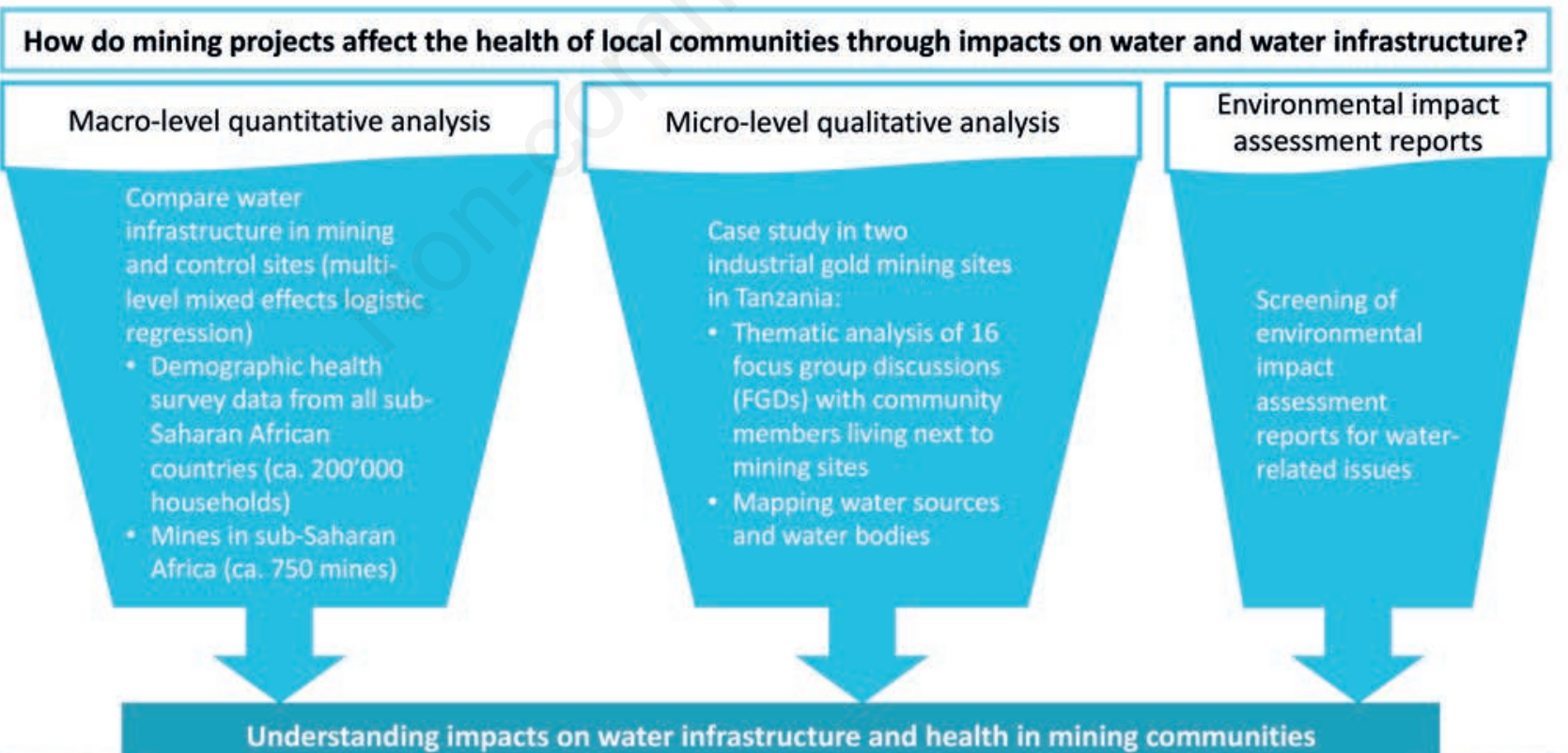

Promote universal access to safe and clean water (SDG 6) through impact assessement

Figure 1. Overview of methodological approach to map water infrastructure and related health impacts in communities affected by mining projects. SDG, Sustainable Development Goal. 


\section{Data triangulation and visualization}

Based on the individual quantitative and qualitative research related to this work, we identified, extracted and combined findings on emerging water and health issues for this data triangulation. Additionally, we contextualised the findings with insights from EIA reports of the two mines in the selected study sites, which were systematically screened for water-related issues. We visualized our findings from the different data sources and levels of data analysis, including spatial components in a short video clip. This approach allowed us to contrast and contextualise our findings from the different levels by portraying the situation on the ground. The main purpose of our visualization was to create a tangible communication tool to present our mixed methods research approach and findings to a broad audience.

\section{Results}

Based on the quantitative data, we found that across all included countries in sub-Saharan Africa, households located closer to a mine were almost four times more likely to have access to piped water, while relying less on surface water. However, stratified analyses revealed that it were mainly the wealthier households that profited from these overall positive trends in the mining regions (Dietler et al., 2021, undergoing peer review).

This information from the DHS data analysis were found to be mirrored by the qualitative data collected in two mining areas in north-western Tanzania (Leuenberger et al., 2021, undergoing peer review). In both study sites, the mine supported the construction of water access points, such as taps, pumps or drilled wells for surrounding communities. However, FGD participants raised concerns about water accessibility. Additionally, we observed several abandoned or not operational pumps, which were originally implemented by mining companies. This confirms the FGD participants' notion on the lack of sustained improvements of water infrastructures. Besides technical aspects, participants from both study sites reported negative impacts of the mining operations on the availability and quality of drinking water from traditional water sources, such as open wells and rain water collection systems. Further, they linked the scarcity and poor water quality of the water with adverse effects on their health status. For example, some FGD participants perceived an increase in diarrhoeal diseases that they linked with water pollution. On the other hand, quantitative data on the incidence of childhood diarrhoea show no association between the location of the mines and incidence of diarrhoeal diseases (Dietler et al., 2021, undergoing peer review). This may be explained by the diversity of factors influencing diarrhoea incidence, including nutritional status, personal hygiene and access to safe drinking water and food.

Screening of the impact assessment reports of the two mining projects revealed that besides various community development initiatives, interventions related to water infrastructures were considered in both mining sites. Both reports included aspects related to water quality and availability in surrounding communities. However, the planned interventions to mitigate these impacts around the Buzwagi mine were more comprehensive compared to the Bulyanhulu mining project. Despite these mitigation plans, our results from the micro-level analyses suggest that communities around Bulyanhulu benefited more from investments in water infrastructure compared to the communities around Buzwagi. This indicates a potential gap between mitigation plans and actual implementations.

\section{Conclusion and outlook}

By combining quantitative and qualitative data, this study highlights differences in impacts of mining companies on water infrastructure and related health effects from a macro- and a microlevel perspective. The triangulation of these different perspectives allowed us to identify pockets in society that did not profit from the overall positive impacts by the mines on water infrastructure. These developments could potentially be driven by direct investments by the mine, but also be the result of overall economic development and increased engagement of the local government in the water sector (Admiraal et al., 2017). Yet, more research is needed to better understand how positive impacts can be maximized by focussing on exposure pathways of local communities to these chemicals. Nevertheless, our results show that mines play a major role for both positive and negative impacts on water availability and quality for local communities and their health status.

This data triangulation was realized within the framework of a larger research project, aiming to generate sound scientific evidence to promote the use of Health Impact Assessment (HIA) in sub-Saharan Africa (Farnham et al., 2020; Winkler et al., 2020). This visualization along with other audio-visual material was produced to communicate the multi-layered research results to a broad audience and facilitate an informed and effective policy dialogue. In particular, this vHealth communication can increase accessibility and raise awareness of public health science in traditionally non-health sectors. Reducing the barriers between different sectors and disciplines can foster intersectoral collaboration between public health practitioners and researchers, international policymakers and local communities, which is key to achieving SDG 3 'Good health and well-being for all' and SDG 6 'Clean water and sanitation' by 2030 .

\section{Motivation}

- Create a tangible communication tool to present our mixed methods research approach and resulting findings to a broad audience;

- visualize and integrate different data sources and levels of data analysis, including geospatial aspects;

- show the different types of water infrastructures commonly found in mining areas on the African continent.

\section{Software used}

- Content visualization and animations: Microsoft Power Point 2016 (Microsoft Corporation, Edmond, WA, USA);

- three-dimensional fly-throughs: Google Earth Pro version 7.3.14507 (Google, Inc., Mountain View, CA, USA);

- video editing: DaVinci Resolve 15 (Blackmagic Design, free download available from: https://www.blackmagicdesign.com /uk/products/davinciresolve/ 
Leuenberger A, Winkler MS, Cambaco O, Cossa H, Kihwele F, Lyatuu I, Zabré HR, Farnham A, Macete E, Munguambe K, 2021. Health impacts of industrial mining on surrounding communities: local perspectives from three sub-Saharan African countries. PLoS One [under review].

Marcantonio RA, Field SP, Sesay PB, Lamberti GA, 2021. Identifying human health risks from precious metal mining in Sierra Leone. Reg Environ Change 21(2) [Epub ahead of print].

Morgan RK, 2012. Environmental impact assessment: The state of the art. Impact Assess Pro A 30:5-14.

Pelders J, Nelson G, 2018. Living conditions of mine workers from eight mines in South Africa. Dev So Afr 36:265-82.

Schrecker T, Birn AE, Aguilera M, 2018. How extractive industries affect health: political economy underpinnings and pathways. Health Place 52:135-47.

United Nations, 2015. Transforming our world: the 2030 Agenda for Sustainable Development. United Nations, New York, NY, USA.

von der Goltz J, Barnwal P, 2019. Mines: The local wealth and health effects of mineral mining in developing countries. J Dev Econ 139:1-16.

Winkler MS, Adongo PB, Binka F, Brugger F, Diagbouga S, Macete E, Munguambe K, Okumu F, 2020. Health impact assessment for promoting sustainable development: the HIA4SD project. Impact Assess Pro A 3:225-32. 